RESEARCH ARTICLE

\title{
Photonic crystal rod-based high-performance ultrafast fiber laser system
}

\author{
Zhiguo $\mathrm{Lv}^{1}$, Zhi Yang ${ }^{2}$, Qianglong $\mathrm{Li}^{2}$, Feng $\mathrm{Li}^{2}$, Yishan Wang ${ }^{2}$, Wei Zhao ${ }^{2}$, and Xiaojun Yang ${ }^{2}$ \\ ${ }^{1}$ School of Physical Science and Technology, Inner Mongolia Key Laboratory of Nanoscience and Nanotechnology, Inner Mongolia \\ University, Hohhot 010021, China \\ ${ }^{2}$ State Key Laboratory of Transient Optics and Photonics, Xi'an Institute of Optics and Precision Mechanics, Chinese Academy of \\ Sciences, Xi'an 710119, China
}

(Received 17 August 2020; revised 16 October 2020; accepted 26 October 2020)

\begin{abstract}
In this paper, we innovatively conduct a Porro prism-based beam pointing stability promotion technique research and realize a high-performance rod-type photonic crystal fiber-based chirped pulse amplification (CPA) system, mainly including a frequency-reduced all-fiber pre-amplification stage, photonic crystal rod-based main amplification stage, and 1600 lines/mm transmission grating-pair compressor. Laser output with average power of $50 \mathrm{~W}$, repetition rates of $500 \mathrm{kHz}$, pulse energy of $100 \mu \mathrm{J}$, pulse duration of $830 \mathrm{fs}$, beam quality of $M^{2}<1.3$, power fluctuation of $0.55 \%$ root mean square, and beam pointing drift of $19 \mu \mathrm{rad} /{ }^{\circ} \mathrm{C}$ over $8 \mathrm{~h}$ is realized. The high-performance laser system has an enormous application potential in fundamental research and precision manufacturing fields.
\end{abstract}

Keywords: chirped pulse amplification; ultrashort pulses; high performance; laser system

\section{Introduction}

In the last few years, ultrafast laser systems have found unprecedented application success in numerous fields of science and industry, e.g., atto-second science, strongfield physics, bio-photonics, and automotive and aerospace machining fields ${ }^{[1-5]}$. For now, most of the femtosecond lasers in practical use are configured in chirped pulse amplification (CPA) architectures based on bulk solid-state crystals or flexible gain fibers. The conventional bulk solidstate laser systems have advantages in achieving pulses with high peak power ${ }^{[6-8]}$ and short pulse duration ${ }^{[9]}$. However, they are also restricted in repetition rate and average power owing to thermo-optical effect induced beam distortion $^{[10-12]}$.

To increase pumping capacity and simultaneously weaken nonlinear effects, single-crystal fiber (SCF) amplifiers have been constructed and demonstrated strong potential for generating pulses with high energy ${ }^{[13,14]}$ and allowing hundreds

Correspondence to: Zhiguo Lv, School of Physical Science and Technology, Inner Mongolia University, Hohhot 010021, China; Xiaojun Yang, Xi' an Institute of Optics and Precision Mechanics, Chinese Academy of Sciences, Xi'an 710119, China. Email: lvzhiguo@imu.edu.cn (Z. Lv); yxj@opt.ac.cn (X. Yang) of average power laser outputs ${ }^{[15]}$. However, in the femtosecond regime, owing to narrow amplification bandwidth and high quantum defect, the efficient femtosecond SCF amplifiers are less convenient to be implemented compared with other ytterbium $(\mathrm{Yb})$-doped gain media ${ }^{[16]}$.

In contrast with conventional bulk solid-state counterparts and SCFs, Yb-doped gain fibers offer a more efficient pumping scheme, diffraction limited beam quality, and broader gain bandwidth ${ }^{[17,18]}$. First, fiber lasers offer a lot of practical advantages over conventional solid-state counterparts, such as reduced alignment sensitivity, compact size, and low cost. In addition, benefiting from the highpower cladding-pumping technique, large single-pass gain characteristic, and flexible propagation route, fiber lasers are acknowledged as one of the most promising and practical powerful tools in numerous application fields. Second, thermal-induced beam distortion is reduced under the high-power pumping condition because of the high surface-to-volume ratio of the fiber geometry, and as a result, excellent spatial mode quality is guaranteed because of the intrinsic waveguide properties. However, amplification of pulses in fiber amplifiers is subjected to low-nonlinearity threshold limitation (such as self-phase modulation, Raman scattering $)^{[19,20]}$. Both effects induce 
pulse fidelity degradation in the time domain and energy transfer in the spectral regime. In ultrafast optics, stretching seed pulses before amplification and recompressing pulses after amplification are common ways to scale the pulse power and energy. In addition, there is also another approach to weaken the nonlinear effects of fiber, and at the same time enlarge the power and energy of femtosecond pulses, that is, to increase the fiber mode field area (MFA) in combination with the use of a relatively short gain section. Yb-doped gain fibers provide efficient pumping at $976 \mathrm{~nm}$ and at least $15 \mathrm{~dB} / \mathrm{m}$ cladding absorption. Therefore, femtosecond fiber optics amplification systems allow for relatively short gain fiber design to reduce nonlinearity to some extent. The most successful attempt of MFA increment is the development and use of rod-type photonic crystal fibers. Exceptional performances in terms of average power and pulse energy have been demonstrated using photonic crystal rod ${ }^{[21-24]}$.

In addition to the excellent beam quality and clean pulse quality, in industrial-scale precision material processing, beam pointing stability of ultrafast fiber laser systems is also crucial. There are some key factors that can worsen beam pointing stability, such as structure deformation induced by heat stress and beam offset introduced by reflective mirrors used in the compressor. Such factors can significantly degrade machining quality and even render the laser cannot be used in practical precision manufacturing, especially for taper-controlled micro-hole manufacturing with tens of micrometer aperture in automotive and aerospace fields. Without adopting any control technique, the general beam pointing stability can be at least $\sim 100 \mu \mathrm{rad} /{ }^{\circ} \mathrm{C}$ or worse. Therefore, efficient heat dissipation in combination with compact insensitive compressor architecture design is crucial for improving beam pointing stability. However, most of the research literature on rod-type fiber femtosecond amplifiers aimed at scaling average power and improving pulse energy, at the same time reducing nonlinear effects and obtaining ultrashort pulse duration. However, research into improving beam pointing stability has been rarely mentioned, such as in Refs. [21-24]. As a result, in order to meet the critical requirements of practical application, in femtosecond CPA laser systems with large dispersion manipulation, how to reasonably design and integrate the optical route of the compressor and simultaneously ensure the promotion of beam pointing stability remains an important research subject and of great significance.

In contrast with the previous reported experimental research on rod-fiber ultrafast laser systems ${ }^{[21-24]}$, in our work, in addition to the general concerned average power, pulse energy, and pulse duration, we have also directed our research towards a beam pointing stability promotion technique. Therefore, we innovatively implemented a research work on improving beam pointing stability performance of high-power femtosecond fiber CPA laser systems, and presented a photonic crystal rod-based high- performance femtosecond fiber CPA laser source. Through the design of a Porro prism (PP)-based folded optical path in the compressor, a maximum pointing drift of $19 \mu \mathrm{rad} /{ }^{\circ} \mathrm{C}$ over $8 \mathrm{~h}$ is obtained. Not only do we realize system integration, such a design of PP-based folded optical route also significantly improves the beam pointing stability of the presented femtosecond fiber CPA laser system. The previously mentioned beam pointing drift of $19 \mu \mathrm{rad} /{ }^{\circ} \mathrm{C}$ meets most of the application requirements of microhole manufacturing with tens of micrometer aperture, and is significant. Herein, in addition to the excellent beam pointing stability $\left(19 \mu \mathrm{rad} /{ }^{\circ} \mathrm{C}\right.$ over $\left.8 \mathrm{~h}\right)$, the presented high-performance femtosecond fiber laser system also has more than $50 \mathrm{~W}$ average power, pulse energy of $100 \mu \mathrm{J}$, and power fluctuation of $0.55 \%$ root mean square (RMS) over $8 \mathrm{~h}$, parameters sufficient to process most hard and fragile materials. Therefore, the presented femtosecond laser system has important practical value in use, and the laser performance defined by these laser parameters can significantly promote the improvement of machining accuracy and quality of materials.

\section{Experimental setup}

The experimental setup for the proposed photonic crystal rod-based high-performance femtosecond fiber CPA laser system is schematically shown in Figure 1. The laser configuration mainly consists of a home-made SESAM modelocked fiber oscillator, a chirped fiber Bragg grating (CFBG), the first pre-amplification stage, an acousto-optic modulator (AOM), the second pre-amplification stage, photonic crystal rod-based main amplification stage, as well as a PP-based transmission grating-pair compressor.

The previously mentioned home-made seed source delivers pulses with $34.56 \mathrm{MHz}$ repetition rates and power around $5 \mathrm{~mW}$. The central wavelength lies in $1032.5 \mathrm{~nm}$ with $13.7 \mathrm{~nm}$ spectral bandwidths. This laser provides sufficient signal for the following temporal stretching and power amplification.

For the first pre-amplification stage, concretely speaking, it consists of a core-pumped preamplifier and a claddingpumped power amplifier. The core-pumped preamplifier has 0.75-m-long Yb-doped gain fiber (Nufern, PM-YSF-HI-HP) and is pumped forward under the pump power of $120 \mathrm{~mW}$. The cladding-pumped power amplifier has 1.2-m-long double-clad (DC) Yb-fiber with $10 \mu \mathrm{m}$ core diameter and $125 \mu \mathrm{m}$ cladding diameter (Nufern, PLMA-YDF-10/125-M). In experiments, multimode pump power is coupled into the cladding of the DC Yb-doped fiber through a PM $(2+1) \times 1$ pump combiner. The combiner has the same input and output fibers with core/cladding diameter of $10 \mu \mathrm{m} / 125 \mu \mathrm{m}$.

In the second pre-amplification stage, in addition to the 0.6-m-long DC Yb-fiber preamplifier (of the same fiber type as in the first pre-amplification stage), 1.15-m-long 


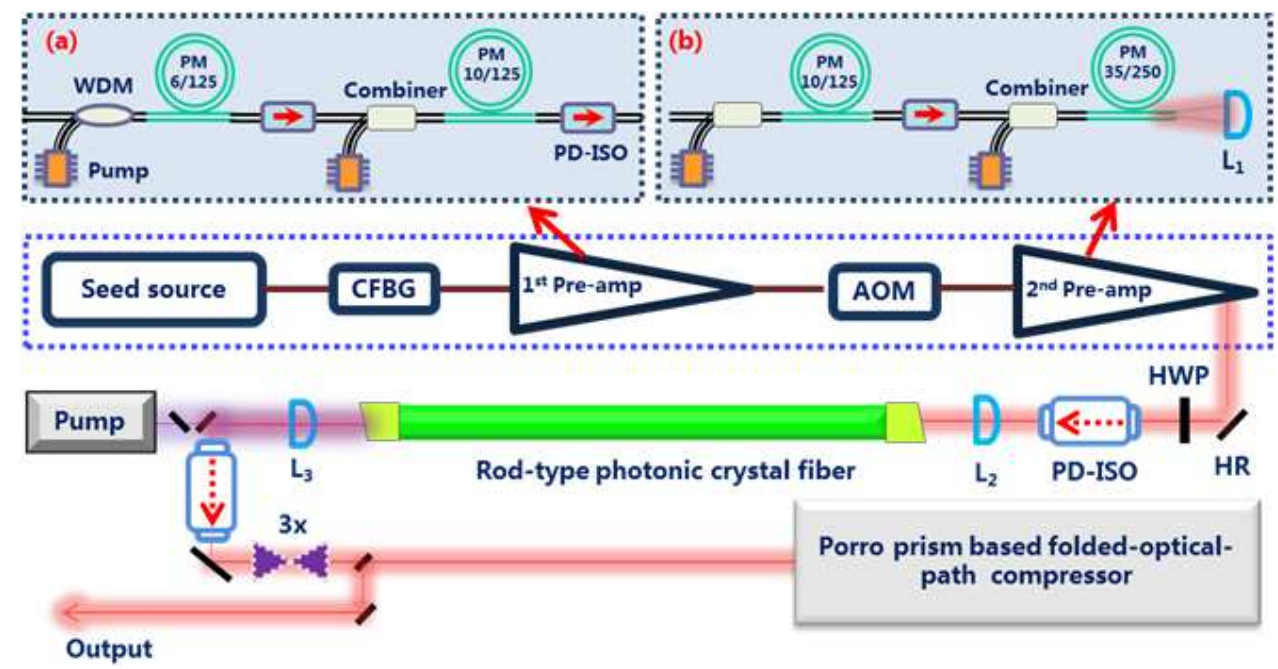

Figure 1. Schematic of the photonic crystal rod-based femtosecond fiber CPA laser system. WDM, wavelength division multiplexer; PD-ISO, polarizationdependent optical isolator; AOM, acousto-optic modulator; CFBG, chirped fiber Bragg grating; HWP, half-wave plate; HR, highly reflective mirror; $\mathrm{L}_{1}$, $\mathrm{L}_{2}$, $\mathrm{L}_{3}$, lens with $30 \mathrm{~mm}, 60 \mathrm{~mm}$, and $20 \mathrm{~mm}$ focal length, respectively.

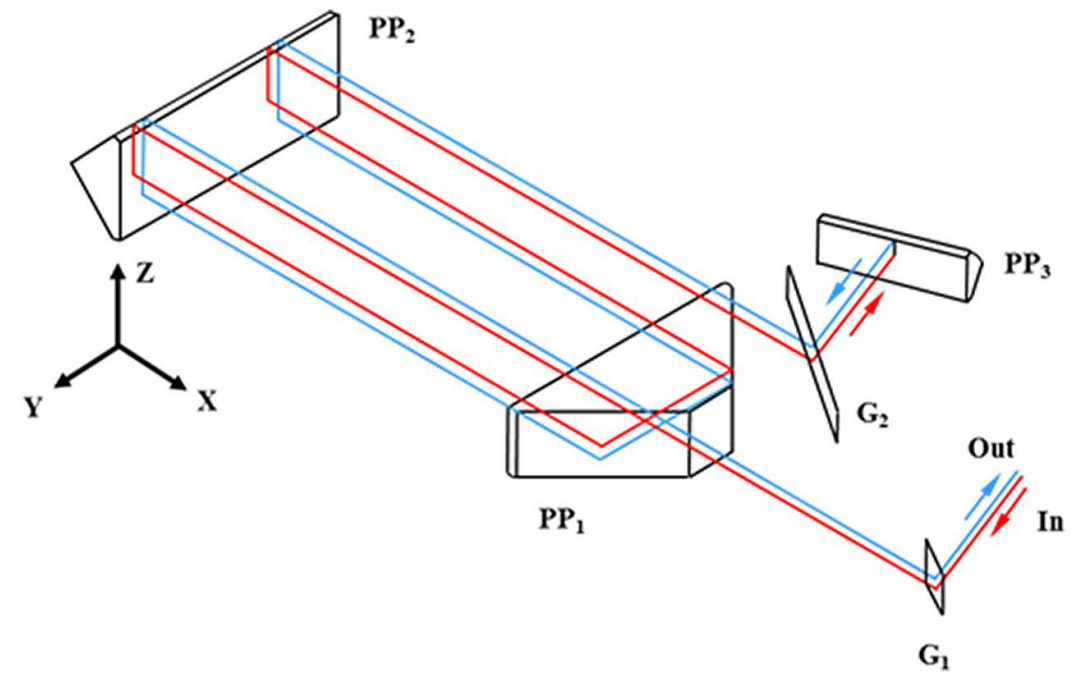

Figure 2. Optical layout of $\mathrm{PP}$-based transmission grating-pair compressor. $\mathrm{G}_{1}$ and $\mathrm{G}_{2}$, gratings; $\mathrm{PP}_{1}, \mathrm{PP}_{2}$, and $\mathrm{PP}_{3}$, Porro prisms.

large-mode-area (LMA) DC Yb-fiber with core/cladding diameter of $35 \mu \mathrm{m} / 250 \mu \mathrm{m}$ (Yb MCOF-35/250-PM) is also employed for further power scaling and energy enhancement. In this stage, two $976 \mathrm{~nm}$ laser diodes with maximum $9 \mathrm{~W}$ and $27.5 \mathrm{~W}$ average power are used as pump sources. Pump power is delivered into the LMA DC fiber through a $(2+1) \times 1$ PM pump combiner with $10 \mu \mathrm{m} / 125 \mu \mathrm{m}$ input fiber and $25 \mu \mathrm{m} / 250 \mu \mathrm{m}$ output fiber.

In the main amplification stage, considering the energydependent nonlinearity, LMA rod-type photonic crystal fiber (NKT, aeroGain-Rod-PM85) is used to weaken the nonlinearity in gain medium as much as possible. The photonic crystal rod has $0.8 \mathrm{~m}$ length and $15 \mathrm{~dB}$ cladding absorption at $976 \mathrm{~nm}$. The core diameter and cladding diameter are $85 \mu \mathrm{m}$ and $260 \mu \mathrm{m}$, respectively. For pumping, a high-power fibercoupled laser diode is used as the drive source and up to
$250 \mathrm{~W}$ continuous wave $(\mathrm{CW})$ power can be emitted. The pump laser from the fiber-coupled laser diode is straight coupled into the photonic crystal rod via an aspherical lens with $20 \mathrm{~mm}$ focal length after being collimated. The collimating aspherical lens has $30 \mathrm{~mm}$ focal length. In experiment, in order to protect the high-power laser diode from damage introduced by the reverse transmitted amplified laser pulses, two orthogonally positioned dichroic mirrors with $99.5 \%$ reflection at $1020-1080 \mathrm{~nm}$ and $94.4 \%$ transmission at 970-980 $\mathrm{nm}$ are inserted between collimating and focusing aspherical lenses.

Finally, to ensure the integration in structure is as compact as possible and simultaneously assure good beam pointing stability, a pointing offset insensitive compressor design has been done based on total internal reflection in PP. The compressor consists of three PPs and a transmission grating 


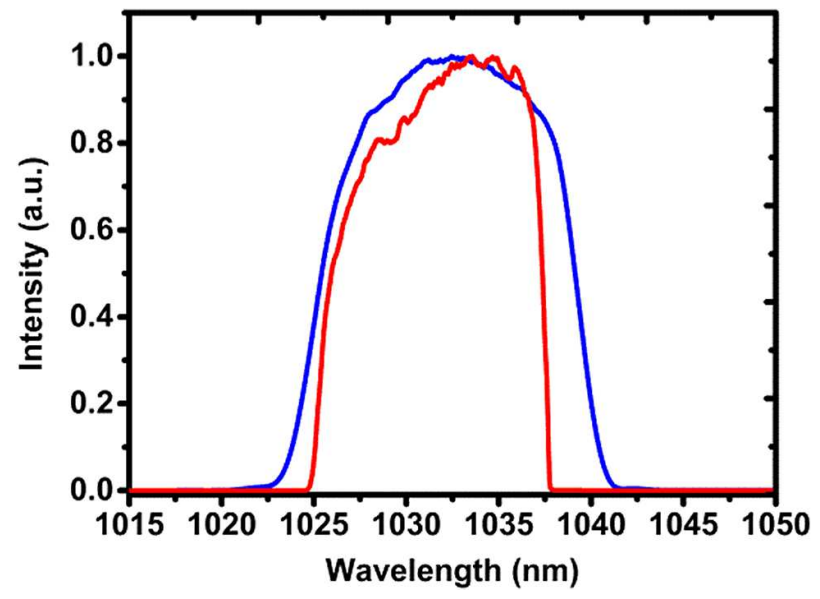

Figure 3. Spectra of the incident seed and stretched pulses with $0.1 \mathrm{~nm}$ resolution. The blue curve shows the spectrum of mode-locked seed pulses and the red curve corresponds to the spectrum of stretched pulses.

pair with 1600 lines $/ \mathrm{mm}$. Figure 2 shows the optical layout of the compressor. The compression chamber dimension is approximately $650 \mathrm{~mm} \times 250 \mathrm{~mm} \times 90 \mathrm{~mm}(L \times W \times H)$. Different from the highly reflective mirror (HR), the design of the pulse compressor is based on total internal reflection in PPs. On the one hand, such a design simplifies the alignment between the incident beam and output beam; on the other hand, it is also insensitive to the offset of incident beam to some extent and potentially improves the output beam pointing stability. In addition, the high line density (1600 lines $/ \mathrm{mm}$ ) and the folded optical route design between PPs realize compactness of the compressor.

\section{Results and discussion}

According to the principle of CPA, the seed pulses need to be stretched in temporal domain before amplification. For the studied laser system, the stretcher consists of a PM circulator, a CFBG, and a home-made temperaturecontrolled module used for dispersion tuning of CFBG. The utilized CFBG has group delay dispersion of $100 \mathrm{ps} / \mathrm{nm}$ and is centered at $1030 \mathrm{~nm}$, and at the same time it also provides reflectivity of more than $70 \%$ and $3 \mathrm{~dB}$ reflection bandwidths of $12 \mathrm{~nm}$. Figure 3 shows the spectra of the incident seed and stretched pulses in blue and red curves, respectively. After stretching, the spectral bandwidths and power of the pulses vary from 13.7 to $11.4 \mathrm{~nm}$ and from 5 to $1.87 \mathrm{~mW}$, respectively.

Next, before reducing the repetition rates to $500 \mathrm{kHz}$, in the first pre-amplification stage, the stretched seed pulses are step-by-step amplified from 1.87 to $24.7 \mathrm{~mW}$ in the corepumped $6 \mu \mathrm{m} / 125 \mu \mathrm{m}$ fiber preamplifier and amplified from 24.7 to $530 \mathrm{~mW}$ in the $10 \mu \mathrm{m} / 125 \mu \mathrm{m}$ cladding-pumped power amplifier, which respectively corresponds to $120 \mathrm{~mW}$ and $1.8 \mathrm{~W}$ pump power. The pulse duration is nearly $1 \mathrm{~ns}$ and the initial peak power of pulses is quite low, which

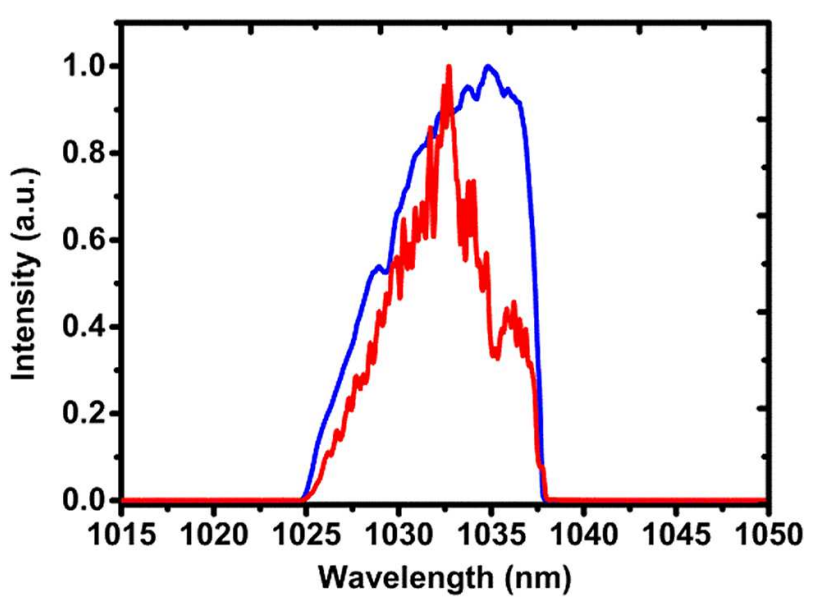

Figure 4. Seed spectrum after reducing the repetition rates to $500 \mathrm{kHz}$ (blue line) and output spectrum of the $35 \mu \mathrm{m} / 250 \mu \mathrm{m}$ LMA DC gain fiber amplification stage (red line).

facilitates realization of high-temporal-quality femtosecond pulses. The spectra after AOM have $1032.5 \mathrm{~nm}$ central wavelength and $9 \mathrm{~nm}$ spectral bandwidths. The spectrum is shown as a blue curve in Figure 4. After the AOM, the average power of $500 \mathrm{kHz}$ pulse source is $4.4 \mathrm{~mW}$. Subsequently, in the second pre-amplification stage, the amplified $500 \mathrm{kHz}$ seed pulses with $81 \mathrm{~mW}$ average power in the 0.6-m-long DC gain fiber preamplifier are further launched into the $35 \mu \mathrm{m} / 250 \mu \mathrm{m}$ LMA DC gain fiber amplification stage, and the average power is boosted to $10 \mathrm{~W}$ level under the pump power of $22 \mathrm{~W}$. The pulse duration is nearly $800 \mathrm{ps}$ and the estimated peak power of pulses is $25 \mathrm{~kW}$. Figure 4 shows output spectrum of the $35 \mu \mathrm{m} / 250 \mu \mathrm{m}$ LMA DC gain fiber amplification stage as a red curve.

In the main amplification stage, the laser delivered from the $35 \mu \mathrm{m} / 250 \mu \mathrm{m}$ gain fiber amplification stage is first collimated using a spherical lens with $30 \mathrm{~mm}$ focal length, and then a spherical lens with $60 \mathrm{~mm}$ focal length is used for coupling the laser into the rod-type PCF to be amplified. To protect the front amplifiers from being disturbed and even damaged through backward propagated laser, highpower polarization-dependent isolators are used between the adjacent gain fiber amplification stages. With reverse pumping as shown in Figure 1, under a pump power of $235 \mathrm{~W}$, the maximum average output power of $97 \mathrm{~W}$ can be achieved. The pulse duration is nearly $600 \mathrm{ps}$ and the peak power of pulses is estimated to be $0.3 \mathrm{MW}$. After the highpower isolator, the degree of polarization of amplified pulses is more than $15 \mathrm{~dB}$. Figure 5 shows almost linearly increased output power versus pump power at $500 \mathrm{kHz}$ repetition rates. The utilized pump source has $976.2 \mathrm{~nm}$ central wavelength and $0.6 \mathrm{~nm}$ spectral bandwidths.

After the main amplifier, the amplified pulses are launched directly into the PP-based pulse compressor in double-pass configuration. As shown in Figure 2, the $\mathrm{PP}_{1}$ and $\mathrm{PP}_{2}$ are inserted between $\mathrm{G}_{1}$ and $\mathrm{G}_{2}$. The incident laser diffracts 


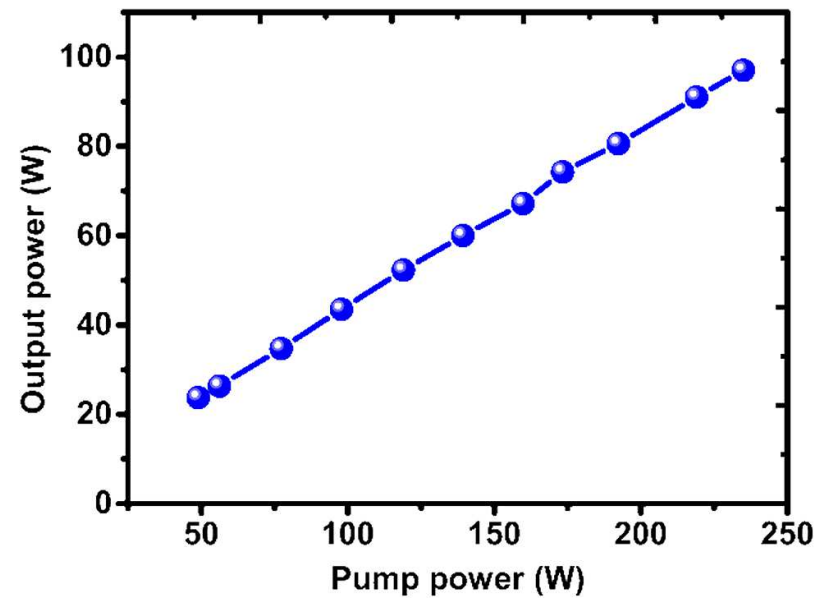

Figure 5. Variation tendency of the amplified average power with the increase of pump power in the photonic crystal rod-based main amplification stage.

efficiently via $G_{1}$ and then propagates between $P P_{1}$ and $\mathrm{PP}_{2}$ via total internal reflection inside of them. Herein, the combination of $\mathrm{PP}_{1}$ and $\mathrm{PP}_{2}$ is employed to fold the optical route so as to realize compact integration. The third prism $\mathrm{PP}_{3}$ is utilized to reflect the laser beam back to the compressor for the next pass through the transmission grating pair. Compared with the conventional combination of reflective mirrors, the combination of PPs has higher structural stability as the propagation direction of output pulses is parallel to that of incident laser pulses all the time due to inherent operation characteristics of PPs. Therefore, such a design of a PP-based folded optical route not only realizes the system integration, but also potentially improves the beam pointing stability of the laser system. For comparison, we calculate the pointing drift for the PP-based compressor architecture and reflective mirror-based compressor scheme, assuming the incident angle remains constant. As shown in Figure 2, the intersecting lines between $\mathrm{PP}_{1}$ and $\mathrm{PP}_{2}$ are perpendicular to each other. As a result, the emergence angle of the output laser beam is insensitive to deviation of both pitching direction of $\mathrm{PP}_{2}$ and deflection direction of $\mathrm{PP}_{1}$, which improves the beam pointing stability. Specifically, if assuming $\mathrm{PP}_{2}$ rotates $0.1^{\circ}$ around the $x$-axis, the output laser beam on incident grating $\mathrm{G}_{1}$ deviates $0.001^{\circ}$ relative to the initial angle, and the corresponding maximum offset for the centroid position of the beam spot is less than $1 \mathrm{~mm}$. However, for the reflective mirror-based compressor scheme, as the reflective mirror varies $0.1^{\circ}$, the corresponding centroid position offset for beam spot is at least $10 \mathrm{~mm}$. Therefore,
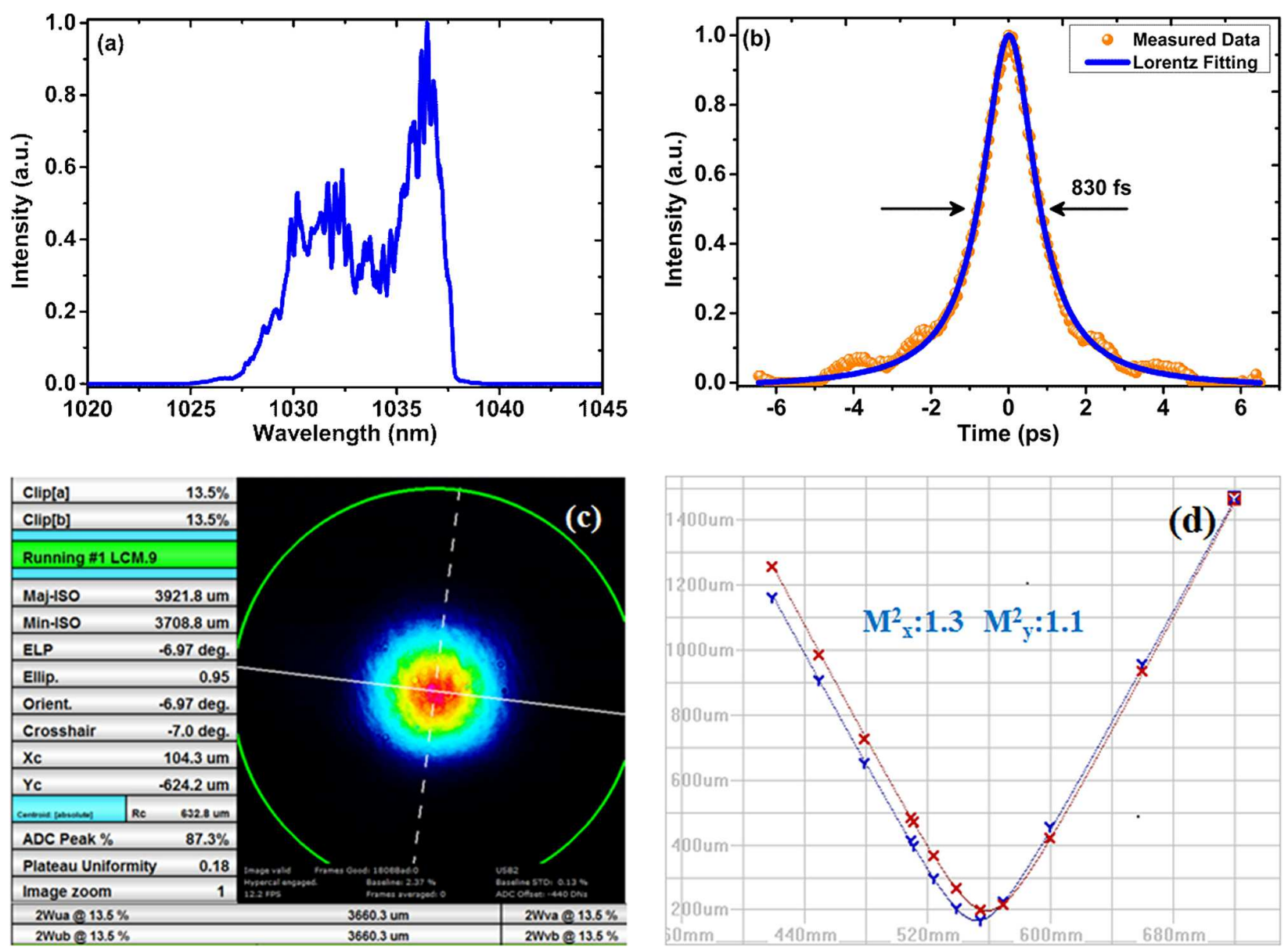

Figure 6. Measured results at $51 \mathrm{~W}$ of compressed average power: (a) optical spectrum; (b) autocorrelation traces; (c) beam profile; (d) $M^{2}$ factors. 

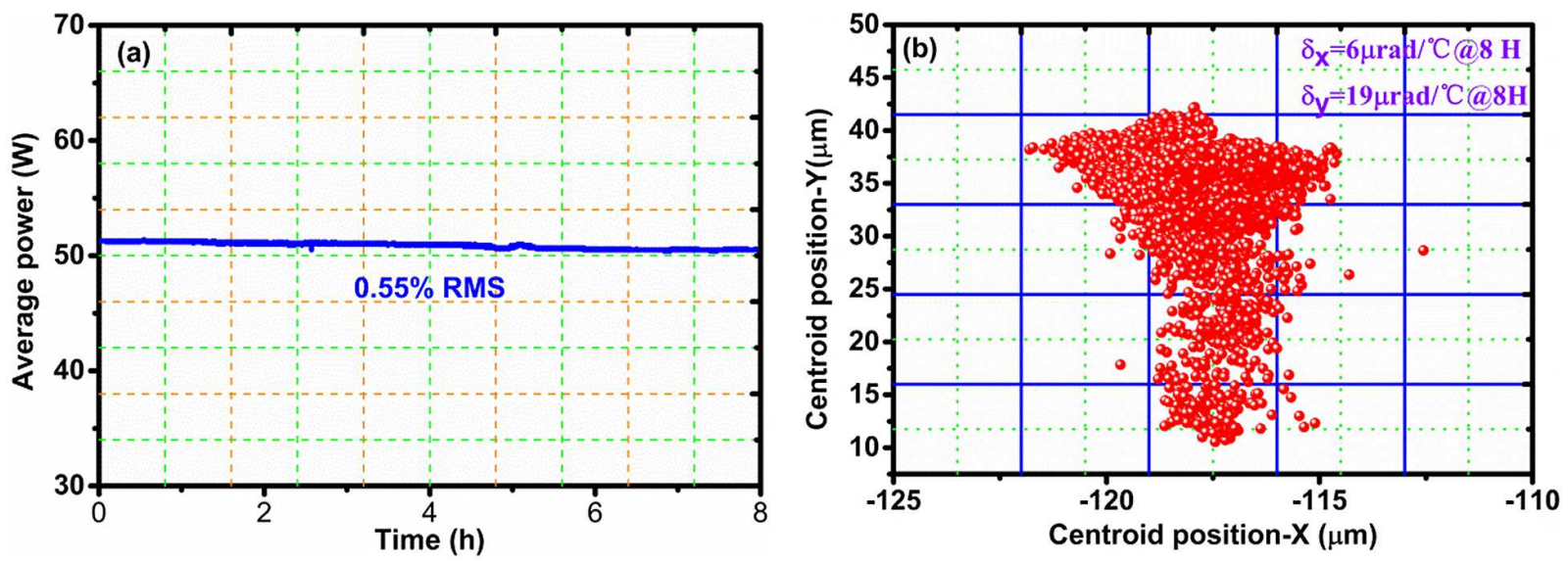

Figure 7. (a) Output power fluctuations and (b) beam pointing stability of the presented laser system in $8 \mathrm{~h}$ at $51 \mathrm{~W}$ of average power and around $100 \mu \mathrm{J}$ single pulse energy.

compared with conventional reflective mirror-based compressor architecture, the PP-based compressor scheme can significantly improve beam pointing stability.

After the compressor, an average power of $51 \mathrm{~W}$ and pulse duration as short as $830 \mathrm{fs}$ are obtainable at 500 $\mathrm{kHz}$ repetition rates, which corresponds to more than 100 $\mu \mathrm{J}$ single-pulse energy. It should be noted that in this laser system, the adopted pump power is actually $150 \mathrm{~W}$. Higher compressible average power output is also obtainable. However, the accumulated nonlinear phase in the main amplifier significantly degrades the pulse fidelity and, thus, limits the compressibility of the amplified pulses. The spectrum of compressed amplified pulses at $51 \mathrm{~W}$ of average power is shown in Figure 6(a). The central wavelength and spectral bandwidths are approximately 1035 and $7 \mathrm{~nm}$, respectively. In comparison with pulse spectra after the $35 \mu \mathrm{m} / 250 \mu \mathrm{m}$ gain fiber amplification stage, the spectral bandwidths after the main amplifier are slightly narrowed owing to the wavelength-dependent gain and gain narrowing effects. In addition, owing to superior heat dissipation and inherent waveguide propagation characteristics, the amplified laser displays excellent beam profile recorded to exhibit a circularity of 95\% and shown in Figure 6(c). As shown in Figure 6(d), the output beam also shows good beam quality with quality factor 1.3 in the horizontal direction and 1.1 in the perpendicular direction. The beam quality is very beneficial for the micro-hole manufacturing of fragile and difficult-to-machine materials.

Further, power fluctuations of the compressed laser pulses have also been measured at $51 \mathrm{~W}$ of average power with the results shown in Figure 7(a). As can be seen, the output power exhibits excellent passive stability of $0.55 \%$ RMS in $8 \mathrm{~h}$. Under the same environmental conditions, we also measure the output beam pointing stability of the presented fiber laser system at $51 \mathrm{~W}$ of average power. With the use of a $500 \mathrm{~mm}$ focal length lens and a beam analyzer, by recording the deviation of centroid position of the focal spot, the measured beam pointing stability is respectively $6 \mu \mathrm{rad} /{ }^{\circ} \mathrm{C}$ in the $x$ direction and $19 \mu \mathrm{rad} /{ }^{\circ} \mathrm{C}$ in the $y$ direction, which is enough for high-precision laser manufacturing. Figure 7(b) shows the measured results of pointing stability in $8 \mathrm{~h}$. Both power fluctuations and beam pointing stability are simultaneously recorded as the temperature varies in a roundtrip period between $22.8^{\circ} \mathrm{C}$ and $26.3^{\circ} \mathrm{C}$. In our work, water-cooling and the PP-based pointing offset insensitive compressor design are the main methods to assure good beam pointing stability.

\section{Conclusion}

In conclusion, we have developed a high-performance femtosecond fiber laser system with compact architecture based on the CPA technique of a photonic crystal rod. The combination of rod-type photonic crystal fiber and PP-based compressor design results in near-diffraction-limited beam quality and high beam pointing stability, while simultaneously preserving the key advantages of fiber laser in terms of compactness, stability, and environmental interference immunity, which are of great concern for high-quality laser precision manufacturing. As a result, we have realized up to $51 \mathrm{~W}$ of average power, and $830 \mathrm{fs}$ pulse duration at $500 \mathrm{kHz}$ repetition rates, which corresponds to around $100 \mu \mathrm{J}$ pulse energy, simultaneously with power fluctuations of $0.55 \%$ RMS in $8 \mathrm{~h}$, pointing stability better than $20 \mu \mathrm{rad} /{ }^{\circ} \mathrm{C}$ in $8 \mathrm{~h}$, and beam quality of $M^{2}<1.3$. The obtainable results provide a powerful base for high-quality applications of femtosecond fiber lasers in precision manufacturing fields.

\section{Acknowledgments}

This work was supported by the Key-Area Research and Development Program of Guangdong Province (No. 2018B090904003), National Natural Science Foundation of 
China (NSFC) (Nos. 61805274 and 6169022), and National Key R\&D Program of China (No. 2018YFB1108000).

\section{References}

1. K. Zhao, Q. Zhang, M. Chini, Y. Wu, X. W. Wang, and Z. H. Chang, Opt. Lett. 37, 3891 (2012).

2. H. Yanagisawa, T. Greber, C. Hafner, and J. Osterwalder, Phys. Rev. B 101, 045406 (2020).

3. W. J. Zong, R. L. Wu, M. L. Li, Y. H. Hu, Y. J. Li, J. H. Li, H. Rong, H. T. Wu, Y. Y. Xu, Y. Lu, H. B. Jia, M. Fan, Z. A. Zhou, Y. F. Zhang, A. M. Wang, L. Y. Chen, and H. P. Chen, Nat. Methods 14, 713 (2017).

4. C. K. Park and D. F. Farson, Int. J. Adv. Manuf. Technol. 83, 2049 (2016).

5. L. Chen, Z. Pan, and J. X. Chen, Opt. Laser Eng. 103, 77 (2018).

6. F. J. Furch, B. A. Reagan, B. M. Luther, A. H. Curtis, S. P. Meehan, and J. J. Rocca, Opt. Lett. 34, 3352 (2009).

7. Z. H. Wang, C. Liu, Z. W. Shen, Q. Zhang, H. Teng, and Z. Y. Wei, Opt. Lett. 36, 3194 (2011).

8. W. Q. Li, Z. B. Gan, L. H. Yu, C. Wang, Y. Q. Liu, Z. Guo, L. $\mathrm{Xu}, \mathrm{M}$. Xu, Y. Hang, Y. Xu, J. Y. Wang, P. Huang, H. Cao, B. Yao, X. B. Zhang, L. R. Chen, Y. H. Tang, S. Li, X. Y. Liu, S. M. Li, M. Z. He, D. J. Yin, X. Y. Liang, Y. X. Leng, R. X. Li, and Z. Z. Xu, Opt. Lett. 43, 5681 (2018).

9. W. L. Tian, C. Yu, J. F. Zhu, D. C. Zhang, Z. Y. Wei, X. D. Xu, and J. Xu, Opt. Express 27, 21448 (2019).

10. S. B. Sutton and G. F. Albrecht, Appl. Opt. 32, 5256 (1993).

11. W. S. Brocklesby, J. Nilsson, T. Schreiber, and J. Limpert, Eur. Phys. J. Special Topics 223, 1189 (2014).
12. T. Eidam, A. Klenke, M. Kienel, S. Breitkopf, L. Grafenstein, J. Limpert, and A. Tünnermann, in CLEO: Science and Innovations (2014), paper JTh4L.5.

13. X. Délen, Y. Zaouter, I. Martial, N. Aubry, J. Didierjean, C. Hönninger, E. Mottay, F. Balembois, and P. Georges, Opt. Lett. 38, 109 (2013).

14. L. Veselis, T. Bartulevicius, K. Madeikis, A. Michailovas, and N. Rusteika, Opt. Express 26, 31873 (2018).

15. V. Markovic, A. Rohrbacher, P. Hofmann, W. Pallmann, S. Pierrot, and B. Resan, Opt. Express 23, 25883 (2015).

16. F. Lesparre, J. T. Gomes, X. Délen, I. Martial, J. Didierjean, W. Pallmann, B. Resan, F. Druon, F. Balembois, and P. Georges, Opt. Lett. 41, 1628 (2016).

17. S. Chénais, F. Druon, S. Forget, F. Balembois, and P. Georges, Prog. Quantum Electron. 30, 89 (2006).

18. Z. G. Lv, H. Teng, L. N. Wang, R. Wang, J. L. Wang, and Z. Y. Wei, Opt. Commun. 370, 156 (2016).

19. H. Kalaycioglu, B. Oktem, Ç. Şenel, P. P. Paltani, and F. Ö. Ilday, Opt. Lett. 35, 959 (2010).

20. A. Fernández, L. Zhu, A. J. Verhoef, D. S. Biryukov, A. Pugzlys, A. Galvabauskas, F. Ö. Ilday, and A. Baltuška, Laser Phys. 21, 1329 (2011).

21. H. J. Otto, F. Stutzki, N. Modsching, C. Jauregui, J. Limpert, and A. Tünnermann, Opt. Lett. 39, 6446 (2014).

22. W. Liu, D. N. Schimpf, T. Eidam, J. Limpert, A. Tünnermann, F. X. Kärtner, and G. Q. Chang, Opt. Lett. 40, 151 (2015).

23. C. P. K. Manchee, J. Möller, and R. J. D. Miller, Opt. Commun. 437, 6 (2019).

24. J. F. Lupi, M. M. Johansen, M. Michieletto, S. L. Christensen, and J. Laegsaard, J. Opt. Soc. Am. B 37, 451 (2020). 